\title{
Early activated hepatic stellate cell-derived paracrine molecules modulate acute liver injury and regeneration
}

\author{
Wenju Chang ${ }^{1,3}$, Lujun Song ${ }^{1,3}$, Xiujuan Chang ${ }^{2,3,4}$, Meiling Ji ${ }^{1}$, Hongshan Wang ${ }^{1}$, Xinyu Qin ${ }^{1}$ and Weixin Niu ${ }^{1}$
}

The effects of paracrine action from early activated hepatic stellate cells (HSCs) on resident liver epithelium cells are not clear. Here, we investigated whether a systemic infusion of early activated HSC-derived paracrine factors (HSC-CM) would evoke an enhanced liver protective response in acetaminophen (APAP)-induced acute liver injury (ALI) in mice and explored the possible underlying mechanisms. The survival rate, liver injury, and liver regeneration were analyzed in mice with or without HSC-CM treatment in vivo. A systemic infusion of HSC-CM provided a significant survival benefit in APAPinduced ALI. HSC-CM therapy resulted in a reduction of hepatocellular death and increased numbers of both proliferating hepatocytes and adult hepatic progenitor cells (AHPCs) with up-regulation of liver regeneration relevant genes. The HSCCM treatment reduced leukocyte infiltration and down-regulated systemic inflammation with decreases in IFN- $\gamma$, IL-1 ra, IL- $1 \beta$, TNF- $\alpha$, and increases in IL-10. The direct anti-death and pro-regeneration effects of HSC-CM on AHPCs were demonstrated using in vitro assays. Treatment with HSC-CM promoted AHPCs proliferation and resulted in increased pAkt expression in vitro, and this effect was abolished by the PI3K/Akt inhibitor LY294002. These data provide evidence that early activated HSC-CM therapy offered trophic support to the acutely injured liver by inhibiting liver cell death and stimulating regeneration, potentially creating a new method for the treatment of ALI.

Laboratory Investigation (2017) 97, 318-328; doi:10.1038/labinvest.2016.130; published online 19 December 2016

Liver transplantation is considered as the only proven, effective treatment for patients suffering from a severe acute liver injury (ALI). However, this therapeutic option is largely limited because of organ donor shortage, high cost, and the requirement for lifelong immunosuppression after transplantation. ALI is usually accompanied by massive hepatocellular death and a compromised capacity for liver regeneration. Therefore, therapies aimed at inhibiting cell death and stimulating endogenous repair pathways would offer major benefits in the treatment of ALI.

Hepatic stellate cells (HSCs) are liver-specific mesenchymal cell types that have vital roles in liver homeostasis and fibrotic liver diseases. ${ }^{1}$ Increasing evidence showed that HSCs have a profound impact on the proliferation, differentiation, and morphogenesis of other hepatic cell types during liver development and regeneration. ${ }^{2,3}$ Activated HSCs have been demonstrated to assist liver regeneration by producing growth factors, which can modulate the proliferation of endothelial cells and hepatocytes, and remodeling the extracellular matrix (ECM). ${ }^{4}$ Inhibiting activated HSCs using gliotoxin ${ }^{5}$ and 1- cysteine $^{6}$ resulted in impaired regenerative responses of both hepatocytes and oval cells in acetaminophen (APAP) and 2AAF/PH-induced ALI models. In addition, $\mathrm{Foxfl}^{+/-}$mice exposed to $\mathrm{CCl}_{4}$ injury had decreased HSCs activation but more severe hepatocyte necrosis during the repair period. ${ }^{7}$ These data established the key functional role of HSCs in the regeneration of the injured liver. However, the mechanisms by which activated HSCs mediate liver regeneration in experimental animals and human patients remain elusive, and the functional roles of various subtypes of HSCs/ myofibroblasts in liver injury are still unclear.

Activated HSCs are known to secrete a number of trophic molecules, including soluble ECM glycoproteins, cytokines, and growth factors. ${ }^{8}$ These factors may directly enhance the proliferation of liver progenitor cells and hepatocytes. Conditioned medium (CM) collected from HSCs harvested from the early regenerating liver of $2 \mathrm{AAF} / \mathrm{PH}$-injured rats contains high levels of HGF, which can promote oval cell proliferation. ${ }^{9}$ Furthermore, HSCs have been shown to have a novel role in liver immunity. ${ }^{10}$ We previously demonstrated

\footnotetext{
'Department of General Surgery, Zhongshan Hospital, Institute of General Surgery, Fudan University, Shanghai, China; ${ }^{2}$ Chinese PLA General Hospital, Medical School of Chinese PLA, Beijing, China and ${ }^{3}$ Treatment and Research Center for Liver Cancer Department, 302 Military Hospital of China, Beijing, China Correspondence: Professor W Niu, Department of General Surgery, Zhongshan Hospital, Institute of General Surgery, Fudan University, 180 Fenglin Road, Shanghai 200032 , China.

E-mail: niu.weixin@zs-hospital.sh.cn.

${ }^{4}$ These authors contributed equally to this work.

Received 31 May 2016; revised 9 October 2016; accepted 6 November 2016
} 
that early activated HSCs (initiation) and persistently activated HSCs (perpetuation) have different responses during an ALI. ${ }^{11}$ However, the underlying mechanism for this process is still unclear; in particular, the local effect of early activated HSCs on resident liver epithelium cells was not fully explored.

In this study, we investigated whether a systemic infusion of early activated HSC-derived paracrine factors (HSC-CM) would lead to a protective liver response during ALI, specifically by inhibiting cell death and stimulating the repair process.

\section{MATERIALS AND METHODS Animals}

Healthy male C57BL/6 J mice (6-8-weeks-old and weighing $20 \pm 2 \mathrm{~g}$ ) were purchased from the Shanghai Laboratory Animal Center at the Chinese Academy of Sciences and were bred under a specific-pathogen-free animal barrier system. The protocols and procedures of animal-use were conducted in accordance with the principles of Laboratory Animal Care (NIH Publication 85-23) and approved by the Ethics Committee of Zhongshan Hospital, Fudan University.

\section{HSC-CM}

HSCs were isolated from healthy male mice, and HSC-CM was prepared as previously described. ${ }^{11,12}$ Briefly, the primary HSCs (approximate yield of $2 \times 10^{6}$ cells per mouse) were cultured for 5 days to induce early activation and were then cultured in Dulbecco's modified Eagle's medium (DMEM) supplemented with $0.05 \%$ bovine serum albumin for $24 \mathrm{~h}$. The medium was collected and concentrated at approximately 25-fold using an ultrafiltration unit (Millipore, Bedford, MA, USA) equipped with a $3-\mathrm{kDa}$ molecular weight cut-off. Finally, the CM, containing paracrine molecules (HSC-CM), was stored at $-80^{\circ} \mathrm{C}$ until use.

\section{ALI Induction and Treatment}

All animals were fasted overnight before APAP (SigmaAldrich, St Louis, MO, USA) treatment. ${ }^{13}$ ALI was induced by an intraperitoneal injection of APAP dissolved in phosphatebuffered saline (PBS) at the dose of $750 \mathrm{mg} / \mathrm{kg}$ body weight. Two hours after the APAP injection, $0.2 \mathrm{ml} 5 \%$ HSC-CM or $0.2 \mathrm{ml}$ serum-free DMEM (vehicle) were infused via the tail vein to treat ALI. Blood samples were obtained at 12, 24, 36, and $48 \mathrm{~h}$ after the injection by a retro-orbital puncture for the analysis of liver enzyme release levels. Mice were killed $24 \mathrm{~h}$ after the injection of APAP, and liver tissues were harvested for the histological analysis. Inactivation of HSC-CM was performed by heating at $100{ }^{\circ} \mathrm{C}$ for $30 \mathrm{~min}$ and immediately cooling on ice.

\section{Assessment of Hepatotoxicity}

The liver injury was evaluated by measuring aminotransferase release in the serum and histological examination of the tissue sections under a light microscope. Alanine aminotransferase
(ALT) and aspartate aminotransferase (AST) were measured using an infinity ALT reagent and an AST reagent, respectively (Thermo Electron, Louisville, CO, USA), according to the manufacturer's instructions. The liver tissues were fixed with $4 \%$ paraformaldehyde in PBS for $18 \mathrm{~h}$ and then embedded in paraffin. Sections of $5 \mu \mathrm{m}$ thickness were cut and stained with hematoxylin and eosin (HE; Sigma-Aldrich). HE-stained liver sections were examined, and necrosis was graded. A histological assessment was performed by a blinded observer, who scored the liver sections using the following criteria: normal histology, '0'; minor hepatocellular death and inflammation, '1'; widely distributed patchy necrosis and inflammation, '2'; complete disruption with panlobular necrosis and inflammation, '3'; and mortality, '4'. Myeloperoxidase (MPO) peroxidation activity was determined in the liver tissue samples with the EnzyFluo Myeloperoxidase Assay Kit, following the manufacturer's instructions (BioAssay system, USA).

\section{Measurements of Serum Cytokine and HSC-CM}

The serum levels of tumor necrosis factor-alpha (TNF- $\alpha$ ), interferon-gamma (INF- $\gamma$ ), IL-6, IL- $1 \beta$, IL-1 receptor antagonist (IL-1ra), HGF, IL-10, MCP-1, MMP2, and MIP- $1 \gamma$ were determined using enzyme-linked immunosorbent assays per the manufacturer's instructions (R\&D Systems, Minneapolis, $\mathrm{MN}$, USA). The serum glutathione (GSH) levels were tested by EnzyChrom GSH/GSSG Assay Kit (BioAssay system, USA).

\section{Histology and Immunohistochemistry}

For histology, HE-stained liver sections were examined, and the necrosis level was graded using a system previously described. ${ }^{14}$ The evaluation of proliferating cell nuclear antigens was performed by bromodeoxyuridine (BrdU) immunohistochemistry. ${ }^{13}$ Briefly, BrdU incorporation was performed by an intraperitoneal injection at a dose of $0.05 \mathrm{~g}$ per gram of body weight $2 \mathrm{~h}$ before harvesting the liver. The evaluation of murine adult hepatic progenitor cell (AHPCs) reactions was performed by Pan-Keratin immunohistochemistry. ${ }^{13}$ AHPCs appeared singly or in irregular strings without lumens in the small portal tracts. To label the apoptotic cells, we performed deoxynucleotidyl transferase-mediated nick-end labeling (TUNEL) staining, for which we used the Fluorescein FragEL DNA fragmentation detection kit (Merck, Darmstadt, Germany) according to the manufacturer's instructions. All sections were examined under Carl-Zeiss microscopy Axiovert 200 (Carl-Zeiss, Jena, Germany) and an image analysis program (AxioVision Ver. 4.0; Carl-Zeiss, Jena, Germany). Nuclei with areas greater than 700 pixel $^{2}$ were analyzed to specifically identify hepatocytes from resident nonparenchymal and infiltrating inflammatory cells. 


\section{Quantitative Real-Time Polymerase Chain Reaction}

Total RNA was isolated from $50 \mathrm{mg}$ of liver tissue using RNAisoTM Plus (TaKaRa, Dalian, China) and was then reverse-transcribed to cDNA using the two-step reverse transcription-polymerase chain reaction (RT-PCR) kit (TaKaRa) and amplified in the ABI 7900HT Fast Real-Time PCR System (Applied Biosystems, Foster City, CA, USA). Gene-specific expression values were normalized to levels of GAPDH within each sample (Supplementary Table 1). Quantitative RT-PCR data were analyzed using the $2^{-\Delta \Delta \mathrm{Ct}}$ method via the SDS 2.1 software, according to the manufacturer's instructions (Applied Biosystems).

\section{AHPCs Isolation and Culture}

AHPCs were isolated by the two-step liver perfusion method by Seglen, with some modifications. ${ }^{15}$ The basic culture medium consisted of modified low-glucose DMEM containing $10 \%$ fetal bovine serum, $10 \mathrm{~mm}$ nicotinamide, $1 \mathrm{~mm}$ Asc2P, $10 \mathrm{ng} / \mathrm{ml}$ epidermal growth factor, insulin-transferring selenium, dexamethasone, streptomycin, and penicillin and was plated on 35-mm culture dishes (BD Falcon, Bedford, MA, USA) coated with rat tail collagen $(50 \mathrm{mg}$ of dried tendon $/ 0.1 \%$ acetic acid) at a low density of $1 \times 10^{4} \mathrm{cells} / \mathrm{cm}^{2}$. The culture conditions were basic medium (vehicle), basic medium mixed at a 20:1 ratio with the 25-fold concentrated HSC-CM (5\% HSC-CM), and at a 5:1 ratio for $20 \%$ HSC-CM.

\section{AHPCs Injury In Vitro}

Forty-eight hours after seeding, AHPCs were incubated with APAP in the culture media as previously described. ${ }^{16}$ AHPCs were cultured in 12-well plates at a density of $1 \times 10^{5} \mathrm{cells} / \mathrm{cm}^{2}$ in a collagen gel sandwich configuration in AHPCs medium. Primary AHPCs were divided into three groups and cultured with the culture medium, 5\% HSC-CM in culture medium and 20\% HSC-CM in the culture medium. All three AHPCs were treated with gradient dozes of APAP $(1.25-20 \mathrm{mM})$ for $24 \mathrm{~h}$ after AHPCs were primary cultured for $48 \mathrm{~h}$. After APAP treatment, cells were rinsed with Hanks' balanced salt solution (HBSS) and incubated with an MTT (3-(4, 5dimethylthiazol 2-yl)-2, 5 diphenyltetrazolium bromide) solution $(1 \mathrm{mg} / \mathrm{ml}$ in HBSS $)$ for $3 \mathrm{~h}$ at $37^{\circ} \mathrm{C}$ to allow the formation of formazan. Thereafter, formazan was solubilized by adding $1 \mathrm{ml}$ of DMSO. Absorbances were measured at a wavelength of $540 \mathrm{~nm}$. APAP toxicity was determined as the $\%$ viability of the treated cells compared with that of untreated cells. Experiments were performed in triplicate.

\section{AHPCs Proliferation In Vitro}

After 12 days of primary culture, AHPCs were cultured with $40 \mu \mathrm{M}$ of the DNA synthesis marker BrdU. After $48 \mathrm{~h}$, the cultures were fixed and stained. The BrdU-positive cells in each cell colony were counted in fluorescence microscopy images. The albumin content in the supernatant samples was determined by enzyme-linked immunosorbent assay using purified rat albumin and a peroxidase-conjugated antibody. The urea content was determined with a commercially available kit (RayBiotech, Norcross, GA, USA), using the manufacturer's instructions.

\section{Western Blotting}

The cultured AHPCs were analyzed using western blotting. Samples were lysed in a detergent lysis buffer. Protein concentrations were measured using a bicinchoninic acid assay (Sigma-Aldrich). Ten micrograms of protein were resolved on a $7.5-10 \%$ SDS-PAGE gel and transferred to nitrocellulose membranes (Millipore, Billerica, MA, USA). The membranes were blocked and incubated overnight at $4{ }^{\circ} \mathrm{C}$ with antibodies against either pAkt, Akt, or GAPDH (1:1000 dilution) and then incubated with a secondary peroxidase-conjugated antibody (1:5000 dilution) after washing. The immunoreactions were visualized using ECL Plus or ECL Western Blotting Detection. The protein expression levels were corrected based on the GAPDH density.

\section{Statistics}

Data are expressed as the mean \pm s.e. of the mean. Statistical significance was determined by a two-tailed Student $t$-test, and a log-rank test Kaplan-Meier curve was conducted for the survival analysis. A $P$-value $<0.05$ was used for statistical significance.

\section{Results \\ HSC-CM Treatment Alleviated Liver Damage and Improved Survival}

Elevated liver enzymes in the peripheral blood provided a good indicator of liver damage. The peak values of the ALT and AST levels in the serum were detected $24 \mathrm{~h}$ after the APAP $(750 \mathrm{mg} / \mathrm{kg}$ ) injection (Figure 1). In the HSC-CMtreated mice, the peak ALT and AST levels were significantly decreased by $42 \%$ and $45 \% \quad(P<0.01)$, respectively (Figures 1a and b), compared with the vehicle-treated mice. In addition, the serum glutathione (GSH) level dramatically decreased after APAP exposure compared with ALI mice treated with HSC-CM or vehicle (Figure $1 \mathrm{~g}$ ). The data further showed that the HSC-CM treatment did not affect GSH levels in the APAP injury model, suggesting that HSC-CM may have a protective effect against liver injury but does not directly influence APAP metabolism.

Previously, we compared the components of the CM from early activated HSCs and persistently activated HSCs and found that early activated HSC-CM contain higher levels of MCP-1, MIP-1 $\gamma$, HGF, MMP2, and IL-10 using a highdensity protein array. ${ }^{11}$ Here, we validated the presence of the high levels of these molecules in HSC-CM by ELISA (Supplementary Figure 1).

During our survival analysis, a significantly improved survival rate was observed for the HSC-CM-treated animals, and this effect was abolished by heat inactivation of HSC-CM (Figure 1c). Only three HSC-CM-treated mice died (3/15) 

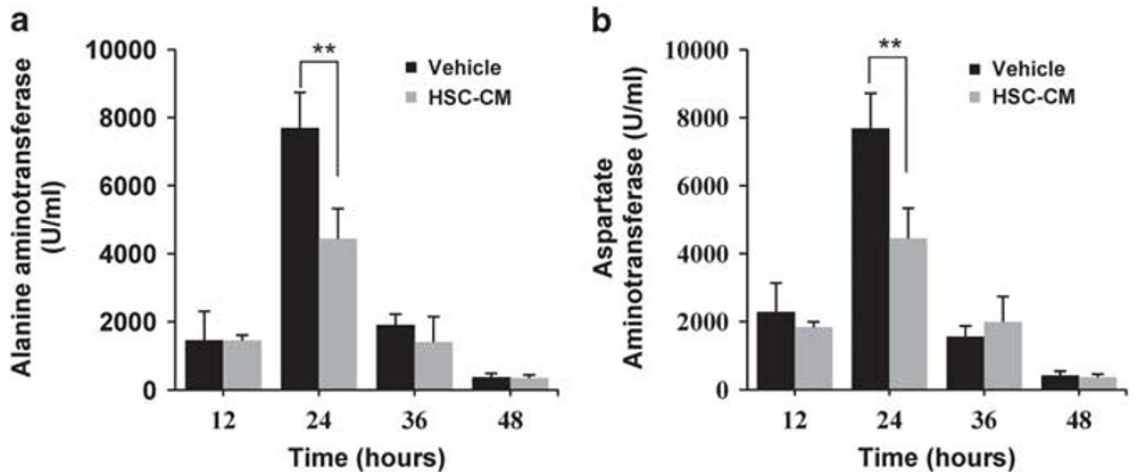

C
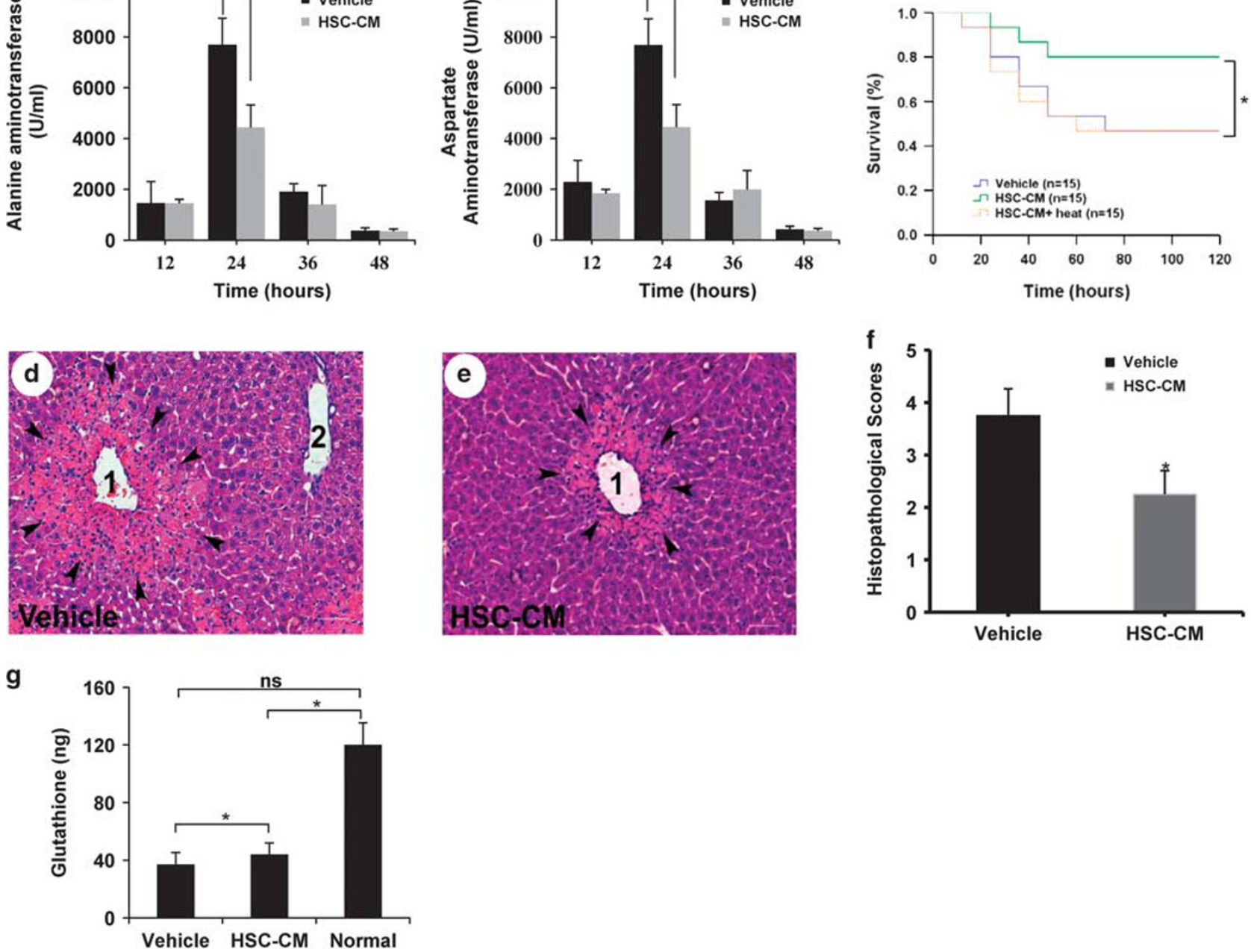

Figure 1 HSC-CM improved hepatocellular necrosis and provided a survival benefit in ALI mice. (a) Alanine aminotransferase and (b) aspartate aminotransferase enzyme levels in peripheral blood samples collected at 12, 24, 36, and $48 \mathrm{~h}$ after the systemic treatment ( $n=4$ per check time point). ${ }^{*} P<0.01$. (c) Kaplan-Meier survival analysis of acetaminophen (APAP)-induced $(750 \mathrm{mg} / \mathrm{kg}$ ) mice treated with concentrated $5 \%$ HSC-CM or heat inactivation of 5\% HSC-CM. Control mice received vehicle. The results for both panels were the cumulative data of two independent experiments $(n=15$ per group). The number of deaths (mice) during survival analysis in each group: 8 in vehicle, 3 in HSC-CM, and 8 in HSC-CM+heat. P-values were determined by the Kaplan-Meier analysis, ${ }^{*} P<0.05$. Microscopic fields of liver tissue were shown after (d) vehicle and (e) HSC-CM treatment $24 \mathrm{~h}$ after APAP injection. Necrotic area was indicated by arrowheads. 1: centrilobular vein, 2: portal vein. Bar $=100 \mu \mathrm{m}$. (f) Scores were determined by semiquantitative histological examination. Data were the mean \pm s.e. of the mean of 10 random high-power fields per animal. (g) Serum glutathione level in APAP-induced ALI. Serum samples were collected from three group animals ( $n=4$ per group) $24 \mathrm{~h}$ after treatment with a systemic injection of $5 \%$ HSC$\mathrm{CM}$, vehicle or normal, healthy mice, and analyzed by a glutathione (GSH) assay kit. Data shown are the mean \pm s.e. of experiments performed in triplicate. ns, not significant; ${ }^{*} P<0.05$. ALI, acute liver injury; HSC-CM, hepatic stellate cell-conditioned medium.

during the experimental period vs 53\% (8/15) of vehicle and HSC-CM heat-treated mice $(P<0.05)$. Altogether, these results showed that the HSC-CM treatment was associated with less severe liver damage and an improved survival rate.

\section{HSC-CM Therapy Improved Microscopic Liver Histopathology}

For the histological analysis, mice were killed, and their liver tissues were harvested $24 \mathrm{~h}$ after the injection of APAP. A microscopic evaluation of HE-stained liver sections revealed hepatocellular death surrounding the central veins (Figures 1d and e), with distorted tissue architecture as previously described. ${ }^{14}$ A semi-quantitative histological examination of the liver tissue confirmed significantly decreased liver necrosis in the HSC-CM group compared with the vehicle group (Figure 1f). The average score in the HSC-CM group was $2.25 \pm 0.45$ compared with $3.75 \pm 0.51$ in the vehicle group $(P<0.01)$. These results demonstrated that the infusion of early activated HSCs mitigated the severity of hepatocellular necrosis. 


\section{TUNEL}

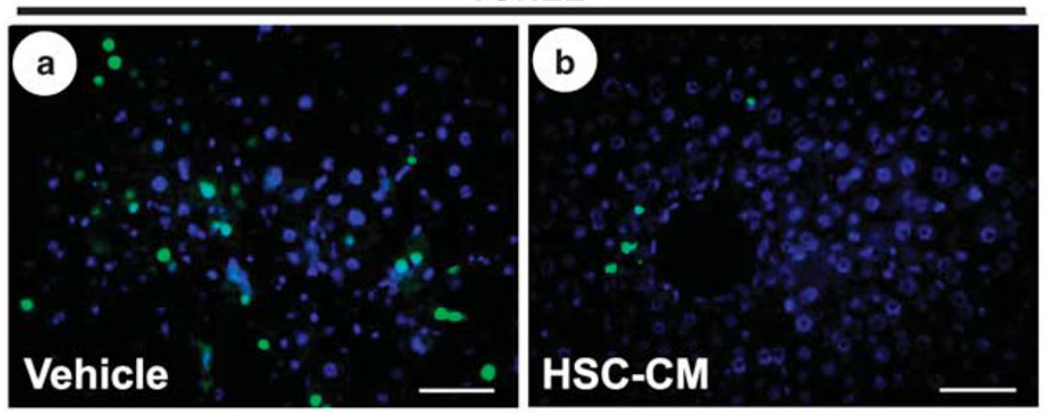

CD45

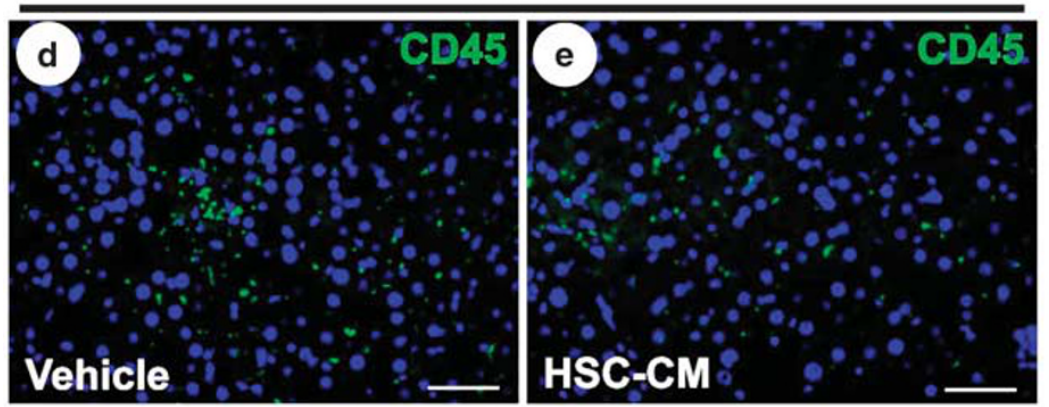

C

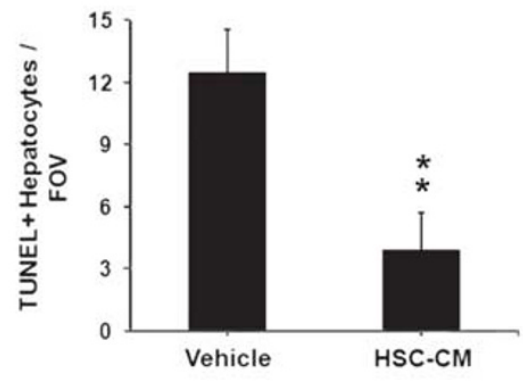

f

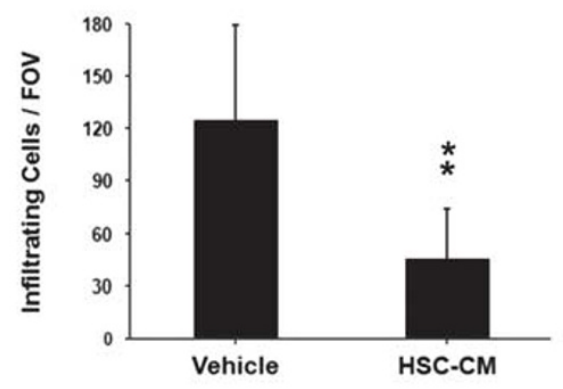

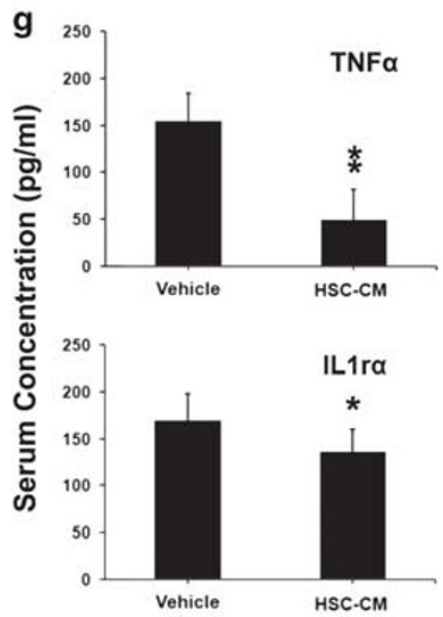
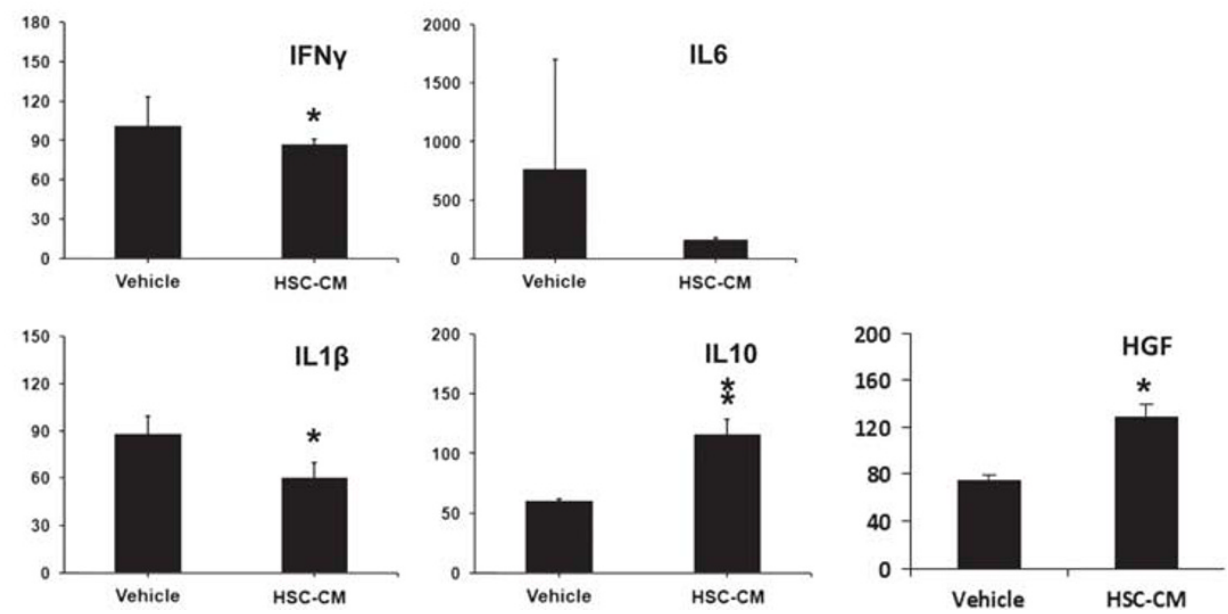

Figure 2 HSC-CM treatment decreased levels of apoptosis and altered systemic inflammatory cytokine profile in acetaminophen (APAP)-induced AlL. ALI mice were killed $24 \mathrm{~h}$ after systemic vehicle or $5 \%$ HSC-CM treatment ( $n=4$ per group). Liver sections were stained by TUNEL (dark brown nuclei, large for hepatocytes) and counterstained with DAPI (light blue). Representative $\times 40$ images from (a) vehicle-treated and (b) HSC-CM-treated mice. (c) Quantification of TUNEL-positive cell nuclei by digital image analysis. Verification of $\mathrm{CD} 45^{+}$infiltrates by immunofluorescence staining from (d) vehicle, or (e) HSC-CM-treated mice. (f) Quantification of infiltrating immune cells. Data shown are the mean \pm s.e. of the mean of 10 random high-power fields per animal. (g) Serum samples were collected from APAP-induced animals $24 \mathrm{~h}$ after treatment with a systemic injection of $5 \%$ HSC-CM ( $n=4)$ or vehicle $(n=4)$, and analyzed by enzyme-linked immunosorbent assay. Data shown are the mean \pm s.e. of experiments performed in triplicate. ${ }^{*} P<0.05$, ${ }^{*} P<0.01$. ALI, acute liver injury; DAPI, 4', 6-diamidino-2-phenylindole; FOV, field of view; HSC-CM, hepatic stellate cell-conditioned medium; HGF, hepatic growth factor; IFN- $\gamma$, interferon-gamma; IL, interleukin; IL-1 ra, IL-1 receptor antagonist; TNF- $a$, tumor necrosis factor-alpha; TUNEL, terminal deoxynucleotidyl transferase-mediated nick-end labeling.

\section{HSC-CM Inhibited Liver Cell Apoptosis In Vivo}

To determine whether HSC-CM infusion affects apoptotic cell death, the number of TUNEL-reactive cell nuclei in the liver sections was determined. Although vehicle-treated mice showed severe pericentral apoptosis (Figure 2a), few apoptotic cells were found in HSC-CM-treated livers (Figure 2b). An
$83 \%$ decrease in TUNEL-positive cell nuclei $(3.93 \pm 1.77 /$ field of view, FOV) was observed following HSC-CM treatment compared with vehicle-treated mice $(23.52 \pm 4.02 / \mathrm{FOV}$; $P<0.01$ ) (Figure $2 \mathrm{c}$ ). These results showed that the HSC$\mathrm{CM}$ treatment correlated with less severe hepatocellular apoptosis. 


\section{HSC-CM Treatment Reduced Leukocyte Infiltration and Down-Regulated Systemic Inflammation}

Severe liver injury results in a local and systemic inflammatory response that can ultimately lead to multi-organ failure and death. We used immunofluorescence to evaluate mononuclear CD45-positive leukocyte infiltration of the liver tissues in the HSC-CM and vehicle groups (Figures $2 \mathrm{~d}$ and e). Liver necrosis with immune cell infiltration was more severe in the livers of vehicle-treated mice. A $64 \%$ decrease in the number of infiltrating CD45-positive immune cells was observed after HSC-CM treatment $(45.58 \pm 28.72 / \mathrm{FOV} v s$ $125.20 \pm 54.05 / \mathrm{FOV}$ in the vehicle group; $P<0.01$; Figure $2 \mathrm{f}$ ). We also tested neutrophils change in the liver tissues of ALI mice with or without HSC-CM treatment by the MPO activity assay and showed that HSC-CM did not affect neutrophils distribution (Supplementary Figure 2).

In addition, the analysis of serum cytokine levels revealed a tendency for IL-6 to decrease with lower levels of IFN- $\gamma(14 \%$; $P<0.05)$, IL-1ra $(21 \% ; P<0.05)$, IL-1 $\beta(32 \% ; P<0.05)$, and TNF- $\alpha(68 \% ; P<0.01)$ in the serum after the HSC-CM treatment (Figure 2g). In contrast, the level of antiinflammatory cytokine, serum IL-10 was increased by 1.9fold $(P<0.01)$, and the HGF concentration was higher $(P<0.05)$ in the HSC-CM-treated animals. Altogether, these data indicated that the infusion of trophic molecules secreted by early activated HSCs reduced leukocyte infiltration and also altered the systemic cytokine profile, which shifted ALI to a more anti-inflammatory state.

\section{HSC-CM Reduced AHPCs Injury In Vitro}

Inhibition of hepatocellular death by HSC-CM therapy in vivo can either be a direct effect of the trophic molecules on preserving AHPCs or an indirect effect, such as via altering the local immunity of the damaged organ. Therefore, we investigated the ability of HSC-CM to directly affect cell death in cultured primary AHPCs. After treatment with APAP $(5 \mathrm{mM})$, the culture medium supplemented with 5\% HSC$\mathrm{CM}$ induced an increase in the cell viability by $23 \%$ compared with the vehicle group $(P<0.01)$ (Figure 3$)$. In contrast, 20\% HSC-CM did not affect cell viability compared with the vehicle group $(P=0.26)$. Overall, these experiments suggested that at a low concentration, HSC-CM had a direct anti-death effect on AHPCs.

\section{HSC-CM Enhanced Liver Regeneration}

Endogenous mature hepatocyte mitosis may represent another potential mechanism of liver regeneration after injury. To determine the effect of HSC-CM treatment on the replication of hepatocytes, a BrdU pulse-chase experiment was performed, and BrdU-positive cells were quantified. We found that more BrdU-positive cells were detected in HSC$\mathrm{CM}$-treated livers (Figure $4 \mathrm{~b}$ ), than in the vehicle-treated mice (Figure 4a). The HSC-CM treatment resulted in a threefold increase in the number of proliferating liver cells compared with the vehicle group $(15.60 \pm 7.19 / \mathrm{FOV}$ vs

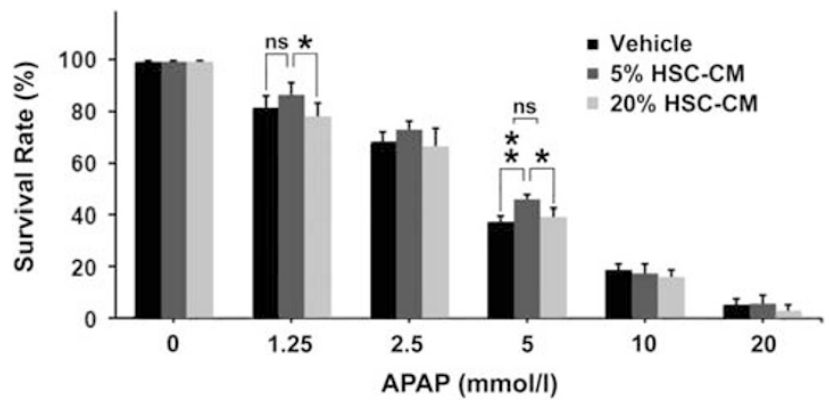

Figure 3 HSC-CM inhibited in vitro adult hepatic progenitor cells (AHPCs) death at low concentrations. Primary AHPCs were cultured in collagencoasted dishes. Cell injury was induced by acetaminophen (APAP) plus into the culture medium. During exposure to APAP, AHPCs were cultured in basic medium only or basic medium supplemented with 5 or $20 \%$ HSC-CM. Primary hepatocytes of the mice were induced with gradient doses of APAP $(1.25,2.5,5,10$, or $20 \mathrm{mM})$ for $24 \mathrm{~h}$. Induction started after $48 \mathrm{~h}$ of cell seeding. Cell viability was determined with MTT cytotoxicity assay. Data are shown as the mean \pm s.e. of the mean. Experiments were performed in triplicate. ${ }^{*} P<0.05,{ }^{*} P<0.01$. HSC-CM, hepatic stellate cellconditioned medium; ns, no significant.

$5.05 \pm 3.14 /$ FOV in the vehicle group; $P<0.05$ ) (Figure $4 \mathrm{c}$ ). In addition, we evaluated AHPCs' reaction in APAP-induced liver injury with or without HSC-CM treatment. AHPCs express phenotypical markers of both immature hepatocyte ( $\alpha$-fetoprotein and albumin) and biliary epithelium lineage (CK7 and CK19, Supplementary Figure 3). AHPCs were defined as cytokeratin-positive cells with oval/cuboidal morphology and a high nuclear to cytoplasmic ratio, which is easily distinguished from the small bile duct with lumens (Figures $4 \mathrm{~d}$ and e). The quantification of AHPCs around the portal tracts with portal veins of cross-sectional areas $<3000 \mu \mathrm{m}^{2}$ showed a statistically significant increase $(P<0.05)$ of AHPC levels after the HSC-CM treatment compared with the vehicle-treated mice (Figures $4 \mathrm{~d}$ and e). In the HSC-CM-treated mice $(n=135$; the number of counted portal tracts), the average number of AHPCs per portal tract was $8.97 \pm 3.75$, and the average number was $7.26 \pm 2.56$ for the vehicle group ( $n=135$, Figure $4 \mathrm{f})$.

We also evaluated the expression levels of seven genes (OSM, HGF, EGF, SCF, IL-6, CK19, and EpCAM) that are known to upregulate during liver regeneration. A quantitative RT-PCR analysis demonstrated that the expression levels of all seven genes were significantly improved (2- to 32-fold) after the HSC-CM treatment (Figure 4g; Supplementary Table 1). These results demonstrated that the HSC-CM treatment improved both the capacity of mature hepatocytes replication and stem cell proliferation.

\section{HSC-CM Stimulated the Proliferation of AHPCs In Vitro}

Liver regeneration is regulated by a complex interaction of paracrine and endocrine signals involving nonparenchymal liver cells. ${ }^{17}$ To determine whether early activated HSCderived molecules can directly enhance AHPC proliferation, 


\section{BrdU}

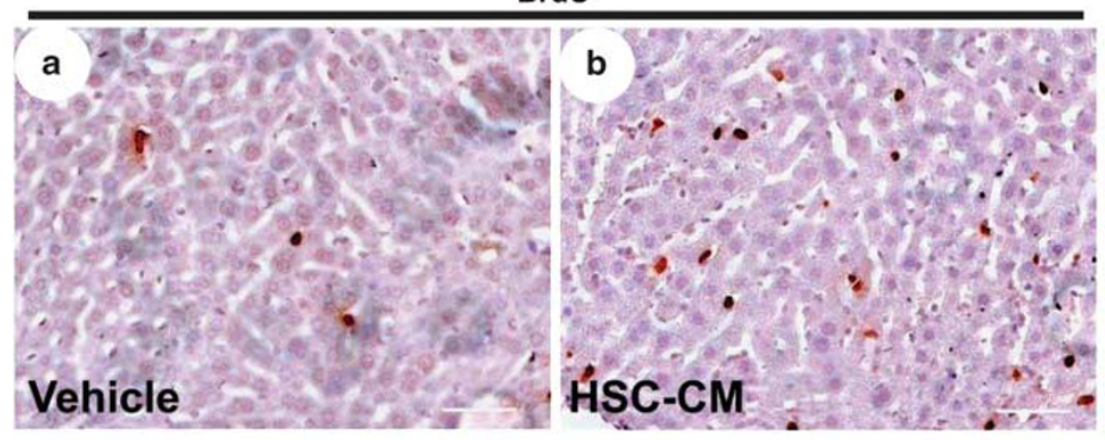

Pank

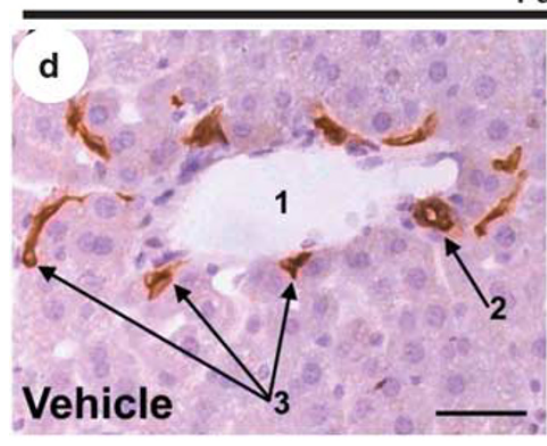

C

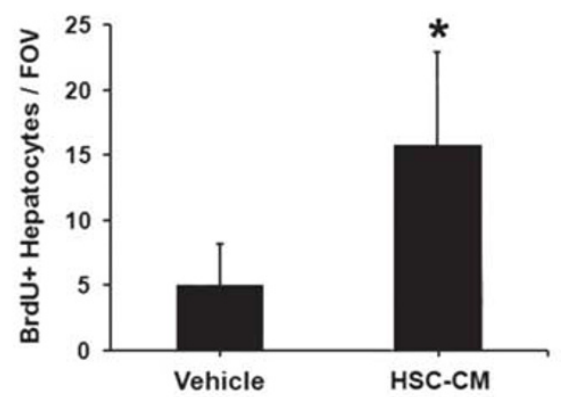

f

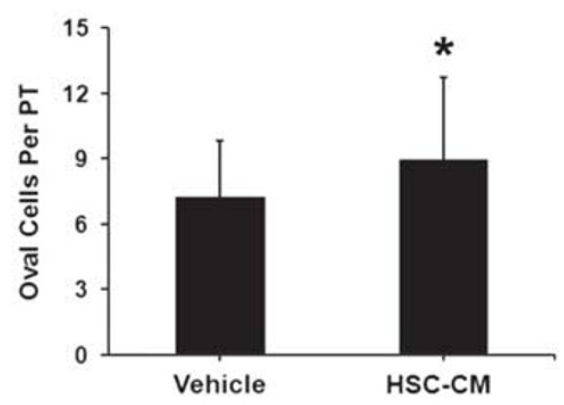

g

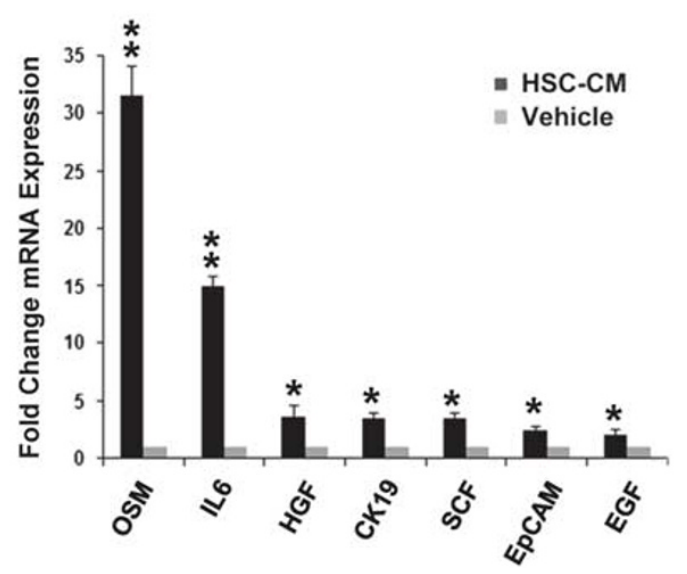

Figure 4 Infusion of HSC-CM enhances liver regeneration in acetaminophen (APAP)-induced ALI in vivo. Liver samples of APAP-induced mice were analyzed $24 \mathrm{~h}$ after treatment with $5 \% \mathrm{HSC}-\mathrm{CM}$ or vehicle. Sections were stained for BrdU (dark brown nuclei). Representative $\times 40$ image from (a) vehicle and (b) HSC-CM-treated animals. Bar $=100 \mu \mathrm{m}$. (c) BrdU-positive hepatocyte-nuclei were quantified by digital image analysis. Data are reported as the mean \pm s.e. of the mean for 10 random fields per animal. Verification of AHPCs by immunofluorescence staining of (d) vehicle, or (e) HSC-CM treatment. 1: Portal vein, 2: small bile duct, 3: AHPCs. (f) Quantification of AHPCs numbers. ${ }^{*} P<0.01$. (g) Quantitative RT-PCR analysis of seven genes known to be up-regulated during liver regeneration, ${ }^{*} P<0.05,{ }^{*} P<0.01$. AHPCs, adult hepatic progenitor cells; BrdU, 5-bromo-2-deoxyuridine; ALI, acute liver injury; CK19, cytokeratin 19; EpCAM, epithelial cell adhesion molecule; FOV, field of view; HSC-CM, hepatic stellate cell-conditioned medium; HGF, hepatocyte growth factor; OSM, oncostatin M; IL-6, interleukin 6; SCF, stem cell factor.

we explored the effect of HSC-CM on AHPCs proliferation in vitro. Compared with Albumin ${ }^{+} / \mathrm{AFP}^{-}$mature hepatocytes (Supplementary Figure 3), AHPCs were $\mathrm{PanK}^{+} / \mathrm{AFP}^{+}$with albumin expression (Supplementary Figure 4).

The proliferation of the AHPC colonies was visualized by double immunofluorescence staining for BrdU and albumin
(Figures 5a-e). A 5\% HSC-CM supplementation induced greater improvements in the colony-forming rate compared with the vehicle group or the $20 \%$ HSC-CM group (Figure 5f). For the BrdU intake study, a $66.12 \%$ increase in BrdU-positive cells was observed $(P<0.01$; Figure $5 \mathrm{~g})$. The $20 \%$ HSC-CM did not obviously affect cell proliferation $(76.10 \pm 14.48 ; P=0.44)$ compared with vehicle. The total 


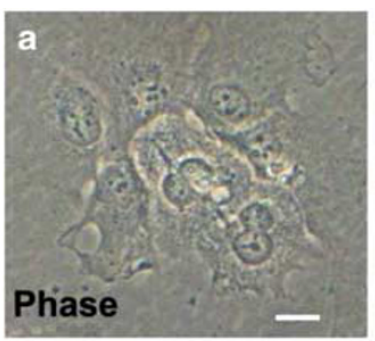

e
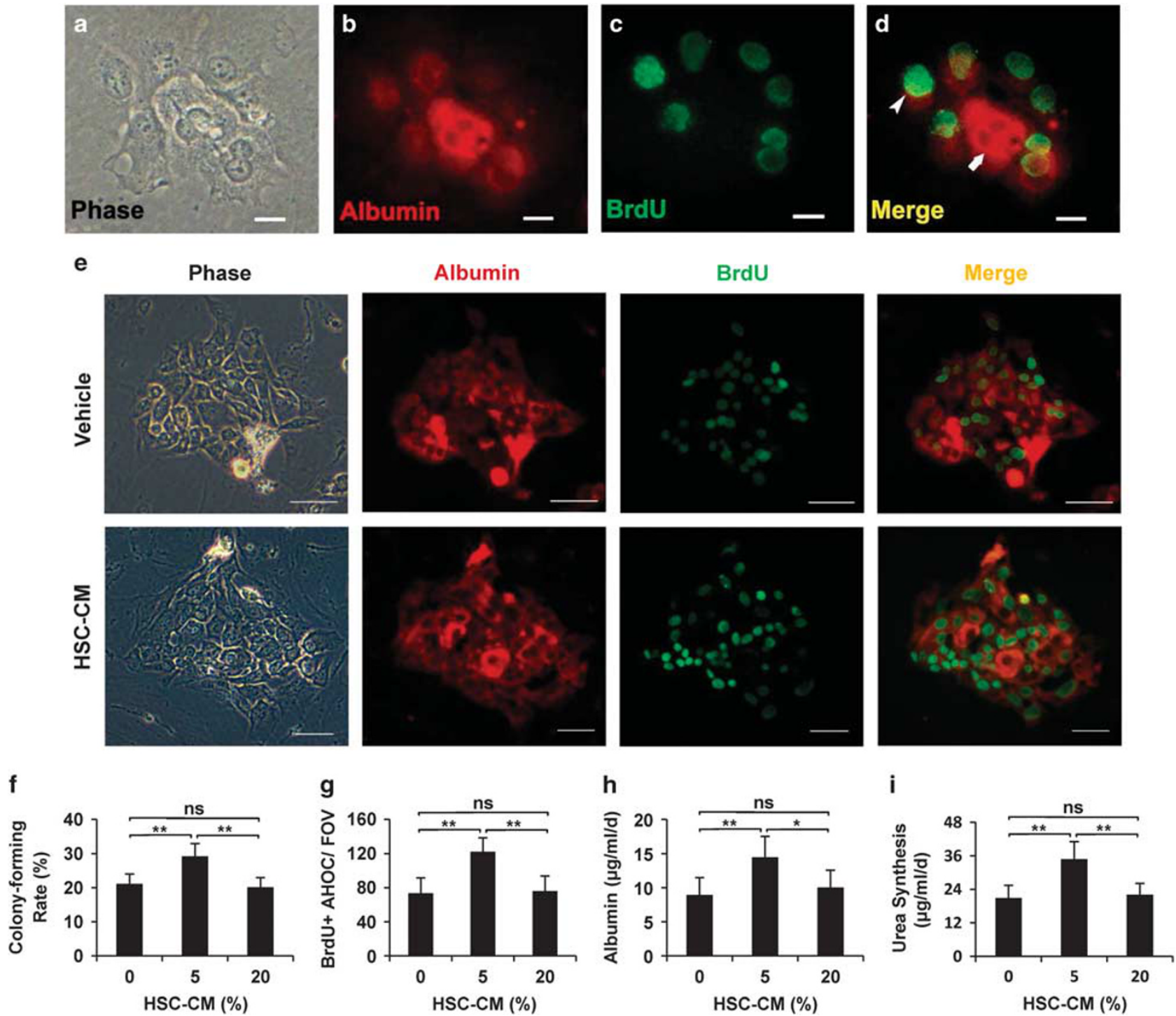

Figure 5 Low concentration of HSC-CM enhanced AHPCs proliferation and cell metabolic functions in Vitro. AHPCs were cultured at low density $\left(1 \times 10^{4}\right.$ cells $/ \mathrm{cm}^{2}$ ) in a dish coated with rat tail collagen. Cells were cultured in basic medium only or basic medium supplemented with $5 \%$ HSC-CM and $20 \%$ HSC-CM. (a-d) Morphology, albumin staining, BrdU uptake, and merged image of proliferating hepatocytes after culture for 4 days with 5\% HSC-CM. (e) Increased hepatocyte colonies size was observed during culture periods, and BrdU uptake study was performed after culture for 14 days. (f)

Quantification of colony-forming rate. (g) Quantification of BrdU-positive hepatocytes. (h) Albumin secretion and (i) urea synthesis test. Data shown are the mean \pm s.e. of two separate experiments in duplicate. ns, not significant; ${ }^{*} P<0.05,{ }^{* *} P<0.01 ; A H P C s$, adult hepatic progenitor cell; BrdU, 5-bromo-2deoxyuridine; HSC-CM, hepatic stellate cell-conditioned medium; FOV, field of view.

amount of albumin secretion and urea synthesis per well was increased in the 5\% HSC-CM group. Albumin levels and urea synthesis were $14.47 \pm 3.04 \mu \mathrm{g} / \mathrm{ml} /$ day and $34.83 \pm 6.21 \mu \mathrm{g} / \mathrm{ml} /$ day in the $5 \%$ HSC-CM treatment compared with $8.93 \pm 2.52 \mu \mathrm{g} / \mathrm{ml} /$ day and $20.87 \pm 4.53 \mu \mathrm{g} / \mathrm{ml} /$ day under the vehicle conditions $(P<0.01$; Figures $5 \mathrm{~h}$ and i). The $20 \%$ HSC-CM group had similar albumin levels to the vehicle group. These data, demonstrating that the effect of HSC-CM was abrogated at a higher concentration (20\%), may suggest a window of therapeutic effectiveness.

\section{HSC-CM Activated Akt Signaling In Vivo and In Vitro}

The signaling pathway underlying HSC-CM's effects on liver regeneration remains unclear. Some studies indicate that Akt signals may have a role. ${ }^{18,19}$ To clarify which signal was activated after the HSC-CM treatment, we tested the expression of Akt and pAkt in the ALI model. Proteins were extracted from liver tissues $24 \mathrm{~h}$ after the HSC-CM or vehicle treatment. We found that the expression level of pAkt was significantly increased in the HSC-CM group compared with the vehicle control. (Figures $6 \mathrm{a}$ and $\mathrm{b}$ ). 
a

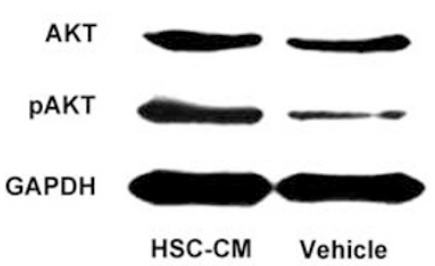

d

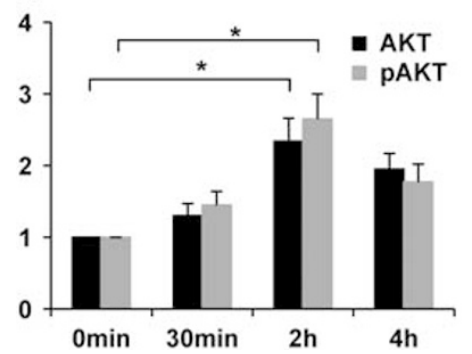

b

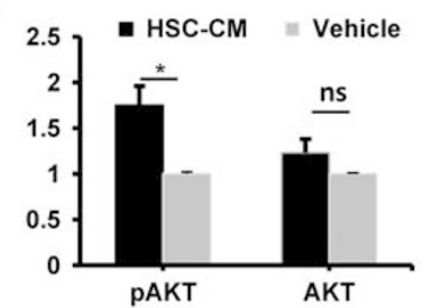

e

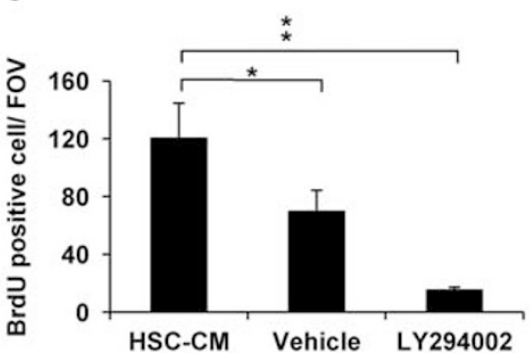

c

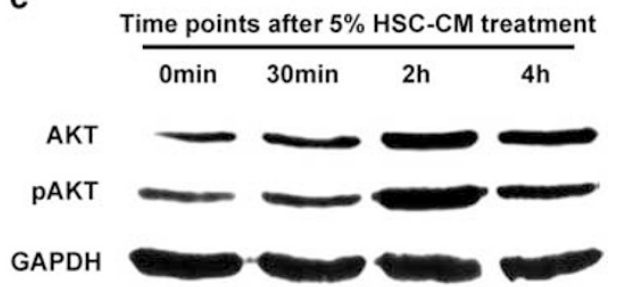

f

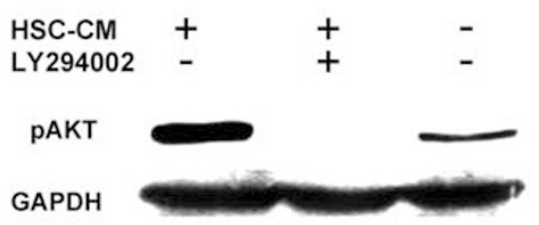

Figure 6 HSC-CM significantly increased the expression of pAkt in vitro and in vivo. (a, b) The expression of phosphorylated Akt (pAkt) and total Akt (Akt) in liver tissues were determined by western blotting $24 \mathrm{~h}$ after HSC-CM treatment. (c) The expression of Akt and pAkt in cultured AHPCs were tested at different time points after in vitro. Isolated AHPCs were cultured for 4 days, after treating with 5\% HSC-CM, Akt and pAKT; protein levels of AHPCs were measured by western blotting analysis. (d) The histogram represents the densitometric analysis of the bands in (c) after HSC-CM stimulation. Similar results were obtained in three independent experiments. (e) LY294002 blocked HSC-CM induced AHPCs proliferation in vitro. The effect of LY294002 on BrdU incorporation in AHPCs was assessed. The AHPCs were divided into three groups; a HSC-CM group, a vehicle, and a HSC-CM plus LY294002 group. In the HSC-CM plus LY294002 group, AHPCs were pre-incubated with LY294002 (50 $\mu$ mol/l) for 30 min, after which 5\% HSC-CM was added in the culture medium. BrdU $(40 \mu \mathrm{mol} / \mathrm{l})$ was added $4 \mathrm{~h}$ before staining, and BrdU-positive cells were calculated. (f) LY294002 specifically blocked PI3K/Akt activation in cultured AHPCs. Three groups of AHPCs were harvested in (e), and pAkt expression levels were compared by western blotting analysis. Similar results were obtained in three independent experiments. AHPCs, adult hepatic progenitor cell; BrdU, 5-bromo-2-deoxyuridine; HSC-CM, hepatic stellate cell-conditioned medium.

To verify the role of Akt signaling in vitro, the expression of pAkt at different time points was examined after adding HSC$\mathrm{CM}$ into the cultured AHPCs. Compared with the untreated group, the expression level of pAkt was significantly increased in the HSC-CM group (Figures $6 \mathrm{c}$ and $\mathrm{d}$ ). To determine the specific role of the Akt signaling pathway in AHPCs' proliferation, the effects of LY294002 (PI3K/Akt inhibitor) on BrdU intake were examined in vitro. The HSC-CM group exhibited significantly increased BrdU-positive cells compared with the vehicle group, and HSC-CM's effect was abolished by a pretreatment with LY294002 $(50 \mu \mathrm{mol} / \mathrm{l})$ (Figure 6e). In addition, the pAkt expression level was compared among these three groups. Pretreatment with LY294002 completely blocked the HSC-CM-mediated Akt phosphorylation as observed by western blotting analysis (Figure $6 \mathrm{f}$ ).

\section{DISCUSSION}

In this study, we provided the first clear evidence that delivery of early activated HSCs secretions (HSC-CM) dramatically reduces cell death and enhances hepatocyte and AHPC proliferation during repair in APAP-induced ALI. Mortality from ALI was partly attributed to the uncontrolled systemic inflammatory response that resulted in multi-organ failure; down-regulation of inflammatory response may contribute to improved survival in ALI. ${ }^{20,21}$ In addition, we found that
HSC-CM treatment led to a down-regulation of proinflammatory cytokines (IL-1ra, IFN- $\gamma$, IL- $1 \beta$, TNF- $\alpha$ ) and an up-regulation of anti-inflammatory cytokines (IL-10), and was associated with locally-reduced leukocyte infiltration in the injured liver. In our previous study, ${ }^{11}$ we performed a protein-array analysis of HSC-CM and detected 69 of 144 assayed proteins, most of which were growth factors, cytokines, and chemokines. Several of the detected molecules, such as IL-10 and HGF, have known antiapoptotic and liver regeneration-stimulating effects. IL-10 is an antiinflammatory pleiotropic cytokine, and endogenous IL-10 protects hepatocytes by suppressing the ability of effector cells (eg, Kupffer cells) to release multiple cytokines, including TNF- $\alpha$ and chemokines, thereby inhibiting cytokinedependent liver injury. ${ }^{20,21}$ After the HSC-CM treatment, increased IL-10 was confirmed in the mice blood by ELISA. These data highly supported the observation that inhibition of the local and systemic immune response resulted in improved survival and a reduction of liver cell death after the HSC-CM treatment. In addition, we demonstrated a direct anti-death effect of HSC-CM treatment on AHPCs after APAP injury in vitro, suggesting that the effect is not limited to the modulation of the inflammatory cascade.

Stimulation of endogenous regeneration mechanisms represents an important method of trophic support by 
HSC-CM therapy in APAP-induced ALI. In the massive injury characteristic of high-dose APAP intoxication, hepatocytes may be unable to accomplish full parenchymal reconstitution; regeneration must include activation and hepatocyte differentiation of HPCs. In animal models of liver regeneration and hepatocarcinogenesis, these HPCs are referred to as oval cells. Kofman et al ${ }^{13}$ confirmed that APAP-induced liver toxicity in the mouse elicits a HPCs response as it does in humans. Moreover, in the earliest period after an APAP challenge, the HPCs reaction is time and dose-dependent. Soeda et al ${ }^{22}$ reported that the betaadrenoceptor agonist, isoproterenol (ISO), was mediated by Wnt signaling and enhanced HPCs expansion to improve mice survival after APAP injury in the short-term. Notably, our results demonstrate that an HSC-CM therapy can increase the proliferative ability of AHPCs and mature hepatocytes after injury, and early activated HSCs secreted factors (such as HGF, one of the most potent stimulants for liver regeneration) are sufficient to accomplish this effect. All biological effects of HGF are mediated by a single tyrosine kinase receptor, c-Met. Gene-knockout studies have shown that both HGF and c-Met are absolutely required for liver development. HGF/c-Met signaling stimulates hepatocyte growth through paracrine and autocrine mechanisms, and these can initiate liver regeneration. ${ }^{23,24}$

Interestingly, the direct stimulatory effect of HSC-CM on the proliferation of AHPCs was abrogated when using a high concentration of HSC-CM in vitro. This suggested that suboptimal HSC-CM therapeutic concentrations may lead to a loss of the therapeutic effect in APAP-induced ALI. Our protein-array analysis of HSC-CM showed that HSC secretions contain a complex mixture of cytokines, growth factors, and chemokines, including small amounts of TNF- $\alpha$, which is a compound with a marked inhibitory effect on liver regeneration. ${ }^{17,25}$ It is possible that, at higher concentrations of HSC-CM, the negative effect of TNF- $\alpha$, for example, overrules the therapeutic effects exerted by the trophic components of HSC-CM. Similarly, the effects of TNF- $\alpha$, present at low concentrations in early activated HSC secretions, become more pronounced at high concentrations of HSC-CM. This may explain why increasing the level of HSC-CM from 5 to $20 \%$ had no added anti-injury effect on AHPCs.

The specific mechanisms of these molecules' actions may be pluralistic, and the signaling pathway involved in HSCCM-induced AHPC proliferation remains unknown. Studies have reported that PI3K/AKT affects early liver regeneration. ${ }^{18,19}$ In this study, we provided pioneering evidence by linking HSCs paracrine action with the activation of Akt signaling in the host. After HSC-CM transplantation into ALI mice, the expression of pAkt was significantly increased in the mouse liver; pAkt, in particular, increased by $\sim 1.8$ times. We also confirmed that HSC-CM could upregulate the expression of pAkt in cultured AHPCs in vitro. Thus, the proliferation of AHPCs was strengthened accordingly. To further confirm that the Akt pathway specifically stimulated AHPCs proliferation after the HSCCM treatment, we observed an inhibition of AHPC proliferation after the Akt pathway was selectively blocked by LY294002. Therefore, activation of the Akt pathway is critical for stimulating the proliferation of AHPCs.

In conclusion, the current study showed that systemic HSC-CM therapy via paracrine mediators reduced hepatocellular death and enhanced liver regeneration, ultimately improving survival in a mouse model of APAP-induced ALI. The PI3K/Akt pathway has an essential role in AHPCs' proliferation after the HSC-CM treatment. This work elucidated that $\mathrm{CM}$ of early activated HSCs induces an integrated response to liver injury and creates potential new avenues for the treatment of ALI.

Supplementary Information accompanies the paper on the Laboratory Investigation website (http://www.laboratoryinvestigation.org)

\section{ACKNOWLEDGMENTS}

We wish to thank Timothy C. Wang and Zhengyu Jiang from Columbia University Medical Center who offered many constructive suggestions for the manuscript revision. This work was supported by grants from the Doctoral Fund of the Ministry of Education (2013007110041), the National Natural Science Foundation of China (81602035), the Young Investigator Funding of Zhongshan Hospital (2014ZSQN37), and the Youth Talent Program of Zhongshan Hospital (2015ZSYXQN10).

\section{DISCLOSURE/CONFLICT OF INTEREST}

The authors declare no conflict of interest.

1. Friedman SL. Hepatic stellate cells: protean, multifunctional, and enigmatic cells of the liver. Physiol Rev 2008;88:125-172.

2. Yin C, Evason KJ, Asahina K, et al. Hepatic stellate cells in liver development, regeneration, and cancer. J Clin Invest 2013;123: 1902-1910.

3. Enami Y, Bandi S, Kapoor S, et al. Hepatic stellate cells promote hepatocyte engraftment in rat liver after prostaglandin-endoperoxide synthase inhibition. Gastroenterology 2009;136:2356-2364.

4. Roskams T. Relationships among stellate cell activation, progenitor cells, and hepatic regeneration. Clin Liver Dis 2008;12:853-860, ix.

5. Shen K, Chang W, Gao X, et al. Depletion of activated hepatic stellate cell correlates with severe liver damage and abnormal liver regeneration in acetaminophen-induced liver injury. Acta Biochim Biophys Sin 2011;43:307-315.

6. Pintilie DG, Shupe TD, Oh SH, et al. Hepatic stellate cells' involvement in progenitor-mediated liver regeneration. Lab Invest 2010;90: 1199-1208.

7. Kalinichenko VV, Bhattacharyya D, Zhou Y, et al. Foxf1 ${ }^{+/-}$mice exhibit defective stellate cell activation and abnormal liver regeneration following CCl4 injury. Hepatology 2003;37:107-117.

8. Balabaud C, Bioulac-Sage P, Desmouliere A. The role of hepatic stellate cells in liver regeneration. J Hepatol 2004;40:1023-1026.

9. Chen L, Zhang W, Zhou QD, et al. HSCs play a distinct role in different phases of oval cell-mediated liver regeneration. Cell Biochem Funct 2012;30:588-596.

10. Chen $\mathrm{CH}$, Kuo LM, Chang $\mathrm{Y}$, et al. In vivo immune modulatory activity of hepatic stellate cells in mice. Hepatology 2006;44:1171-1181.

11. Chang WJ, Song LJ, Yi T, et al. Early activated hepatic stellate cellderived molecules reverse acute hepatic injury. World J Gastroenterol 2015;21:4184-4194.

12. Chang $W$, Yang $M$, Song $L$, et al. Isolation and culture of hepatic stellate cells from mouse liver. Acta Biochim Biophys Sin 2014;46:291-298. 
13. Kofman AV, Morgan G, Kirschenbaum A, et al. Dose- and timedependent oval cell reaction in acetaminophen-induced murine liver injury. Hepatology 2005;41:1252-1261.

14. Liu ZX, Han D, Gunawan B, et al. Neutrophil depletion protects against murine acetaminophen hepatotoxicity. Hepatology 2006;43:1220-1230.

15. Song $\mathrm{L}$, Wang $\mathrm{H}, \mathrm{Gao} X$, et al. Proliferation and differentiation potential of mouse adult hepatic progenitor cells cultured in vitro. Acta Biochim Biophys Sin 2010;42:122-128.

16. Jemnitz K, Veres Z, Monostory $\mathrm{K}$, et al. Interspecies differences in acetaminophen sensitivity of human, rat, and mouse primary hepatocytes. Toxicol In Vitro 2008;22:961-967.

17. Taub R. Liver regeneration: from myth to mechanism. Nat Rev Mol Cell Biol 2004;5:836-847.

18. Zhang $R$, Zhang $L$, Manaenko A, et al. Helium preconditioning protects mouse liver against ischemia and reperfusion injury through the $\mathrm{PI} 3 \mathrm{~K} /$ Akt pathway. J Hepatol 2014;61:1048-1055.

19. Jackson LN, Larson SD, Silva SR, et al. PI3K/Akt activation is critical for early hepatic regeneration after partial hepatectomy. Am J Physiol Gastrointest Liver Physiol 2008;294:G1401-G1410.
20. Bourdi M, Masubuchi Y, Reilly TP, et al. Protection against acetaminophen-induced liver injury and lethality by interleukin 10: role of inducible nitric oxide synthase. Hepatology 2002;35: 289-298.

21. Wang SC, Ohata M, Schrum L, et al. Expression of interleukin-10 by in vitro and in vivo activated hepatic stellate cells. J Biol Chem 1998;273:302-308.

22. Soeda J, Mouralidarane A, Ray S, et al. The beta-adrenoceptor agonist isoproterenol rescues acetaminophen-injured livers through increasing progenitor numbers by Wnt in mice. Hepatology 2014;60: 1023-1034.

23. Ishikawa T, Factor VM, Marquardt JU, et al. Hepatocyte growth factor/cmet signaling is required for stem-cell-mediated liver regeneration in mice. Hepatology 2012;55:1215-1226.

24. Huh CG, Factor VM, Sanchez A, et al. Hepatocyte growth factor/c-met signaling pathway is required for efficient liver regeneration and repair. Proc Natl Acad Sci USA 2004;101:4477-4482.

25. Malhi H, Gores GJ. Cellular and molecular mechanisms of liver injury. Gastroenterology 2008;134:1641-1654. 